\title{
Type I Interferon Signature in Chilblains Following SARS-CoV-2 mRNA Vaccine: A Case Report
}

Karim SOUAID ${ }^{1,2}$, Bénédicte OULÈS ${ }^{1-3}$, Pierre SOHIER ${ }^{2,4}$, Lydia DESCHAMPS ${ }^{5}$, Sélim ARACTINGI ${ }^{1-3 \# *}{ }^{*}$ and Nicolas DUPIN ${ }^{1-3 *}$ ${ }^{1}$ Department of Dermatology, Hôpital Cochin, AP-HP, AP-HP Centre-Université de Paris, 89 rue d'Assas, FR-75006 Paris, ${ }^{2}$ University of Paris, Faculté de Médecine Paris Centre Santé, ${ }^{3}$ Cutaneous Biology Laboratory, Institut Cochin, INSERM U1016, UMR8104, ${ }^{4}$ Department of Pathology, Hôpital Cochin, AP-HP, AP-HP Centre-Université de Paris, and ${ }^{5}$ Department of Pathology, Hôpital Bichat, AP-HP, AP-HP Nord, Paris, France.*E-mail: selim.aractingi@gmail.com

\#These authors contributed equally to this work as co-senior authors.

Accepted Aug 13, 2021; Epub ahead of print Aug 16, 2021

An outbreak of chilblain-like lesions has been reported during the COVID-19 pandemic (1). The mechanism of this phenomenon remains unclear, as detection of SARS-CoV-2 by PCR and serology was negative in most of the patients who presented with chilblain-like lesions and were tested (2). However, authors have suggested that chilblain-like lesions may be the consequence of a viral-induced type I interferonopathy $(3,4)$. SARS$\mathrm{CoV}-2$ may robustly trigger the expression of interferon (IFN)-inducible genes in susceptible individuals, leading to an early IFN-I response, inhibiting viral replication, inducing immune-mediated changes, and subsequently eliciting chilblains-like lesions (5). We report here an original case of a patient developing de novo chilblains following the SARS-CoV-2 vaccine with evidence of IFN signature in lesional skin.

\section{CASE REPORT}

A 55-year-old previously healthy man, with no past medical history, presented at our department with chilblains following the second injection of the SARS-CoV-2 mRNA Pfizer-BioNTech vaccine (tozinameran, Comirnaty). Different cutaneous manifestations began 4 days after receiving the first dose of the vaccine, when he noted 2 solitary indurated nodules on the posterior neck and left clavicle. These lesions were not associated with systemic symptoms and disappeared spontaneously within few days. Five days after the second injection, performed 4 weeks later, he noted 2 painful papular lesions on the dorsum of his second right finger (Fig. 1A), which progressed rapidly into a purpuric well-defined plaque typical of chilblains (Fig. 1B). Skin biopsy showed features of idiopathic chilblains (Fig. 1C). Blood cell count, liver and kidney tests, and inflammatory parameters were unremarkable. In addition, we performed an exhaustive analysis of several biological parameters, including cryoglobulin, anti-nuclear antibodies, anti-neutrophil cytoplasmic antibodies, anticardiolipin antibodies and antiphospholipid antibodies, which showed no abnormalities except a modest increase in antiphospholipid IgG antibodies. Therefore, no other cause of chilblains was identified. The patient was given topical steroids and the lesion disappeared within 7 days. After 2 months of follow-up, there was no relapse.

There was a high level of expression of myxovirus resistance protein $\mathrm{A}(\mathrm{MxA})$ protein in lesional skin immune infiltrate (Fig. 2). The human MxA is known to be a key mediator of the IFN-induced antiviral response against a wide range of viruses. Its expression is tightly regulated by type I IFNs. On the other hand, systemic IFNa level was within the normal range in the current patient, indicating locally increased IFN type I pathway activation.

\section{DISCUSSION}

Clinical heterogeneity of SARS-CoV-2-induced reactions has been observed. While most patients may be asymptomatic, others will develop a cytokine storm leading to acute respiratory distress syndrome. Even if the precise pathophysiology has not yet been fully elucidated, recent reports have indicated that chilblains are likely to be triggered by an exacerbated innate IFNmediated immune reaction targeting viral antigens (3-5), and remain associated with a good prognosis (1).

This case report provides a clinical and histopathological description of chilblains following the administration of the second injection of the Pfizer-BioNTech vaccine. This clinical event, occurring in a patient with

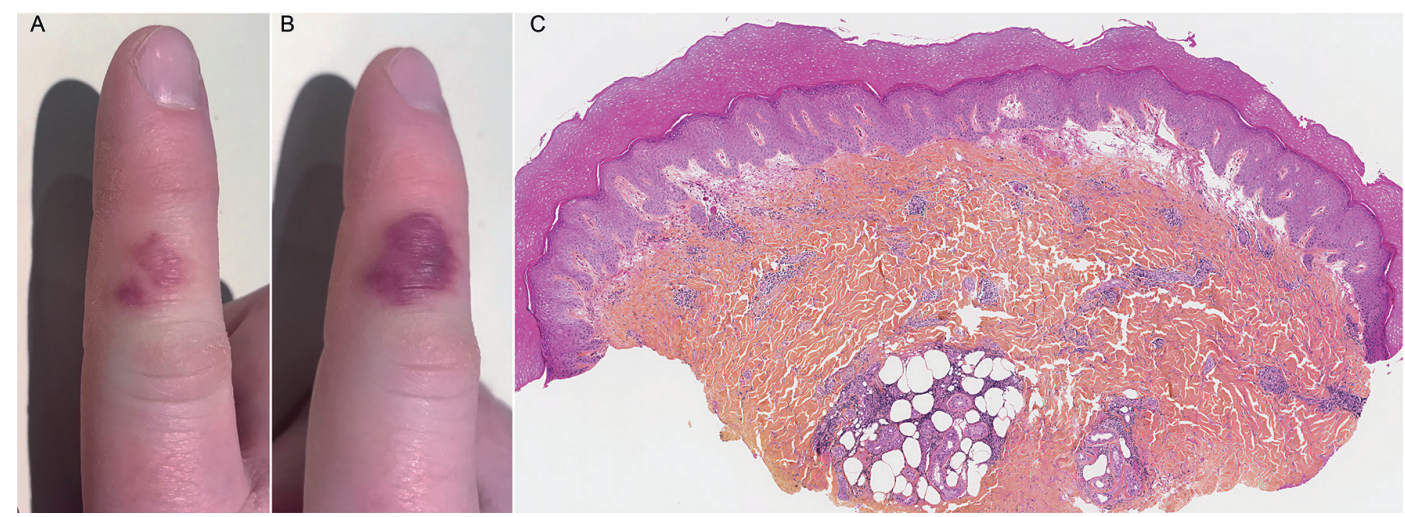

Fig. 1. Clinical and pathological lesions observed in the patient. (A, B) Clinical photographs of chilblains after the second injection of Pfizer-BioNTech mRNA vaccine. (C) Skin biopsy of the lesion showing a perivascular and peri-eccrine lymphocytic infiltrate associated with oedema of the papillary dermis. Hematoxylin-eosin (H\&E) staining, original magnification $\times 5$. 


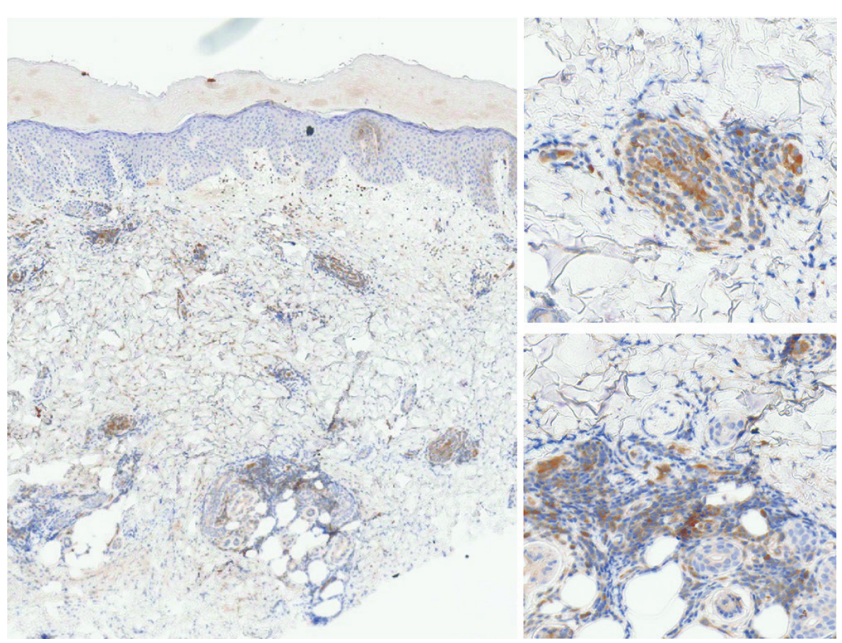

Fig. 2. Myxovirus resistance protein A (MxA) staining of lesional skin. Representative images of MxA staining of lesional skin. Left hand panel: Original magnification $\times 5$, Right hand panel: Original magnification $\times 20$

no past history of auto-immune or vascular disease, suggests a probable attributability of SARS-CoV-2 vaccine, as also proposed by other authors (6). Intriguingly, the occurrence of chilblains in the context of COVID-19 or following SARS-CoV-2 vaccination may indicate a common mechanism likely to involve immune response targeting Spike RNA or protein. In addition, a high level of expression of MxA protein was found in lesional skin, suggestive of locally increased IFN type I pathway activation, as also observed in COVID-19-associated chilblains (3). Additional scientific studies are needed to elucidate the underlying mechanisms.

\section{ACKNOWLEDGEMENTS}

The authors are grateful to all members of the Dermatology Department of Cochin Hospital for helpful discussions, and to Dr Jean-Francois Meritet for constant help and advice.

BO reports congress grants from Novartis and BMS, outside the submitted work. SA reports grants from Novartis and Leo Pharma, and personal fees from Pierre Fabre, BMS, and Lilly, outside the submitted work. The other authors have no conflicts of interest to declare.

\section{REFERENCES}

1. Matar $S$, Oulès B, Sohier $P$, Chosidow O, Beylot-Barry $M$, Dupin $\mathrm{N}$, et al. Cutaneous manifestations in SARS-CoV-2 infection (COVID-19): a French experience and a systematic review of the literature. J Eur Acad Dermatology Venereol 2020; 34: e686-e689.

2. Le Cleach L, Dousset L, Assier H, Fourati S, Barbarot S, Boulard C, et al. Most chilblains observed during the COVID-19 outbreak occur in patients who are negative for COVID-19 on polymerase chain reaction and serology testing*. $\mathrm{Br} \mathrm{J}$ Dermatol 2020; 183: 866-874.

3. Aschoff R, Zimmermann N, Beissert S, Günther C. Type I interferon signature in chilblain-like lesions associated with the COVID-19 pandemic. Dermatopathology 2020; 7: 57-63.

4. Hubiche T, Cardot-Leccia N, Le Duff F, Seitz-Polski B, Giordana $P$, Chiaverini $C$, et al. Clinical, laboratory, and interferonalpha response characteristics of patients with chilblain-like lesions during the COVID-19 pandemic. JAMA Dermatol 2021; 157: 202-206.

5. Sohier P, Matar S, Meritet J-F, Laurent-Roussel S, Dupin N, Aractingi S. Histopathologic features of chilblainlike lesions developing in the setting of the coronavirus disease 2019 (COVID-19) pandemic. Arch Pathol Lab Med 2021; 145: 137-144.

6. McMahon DE, Amerson E, Rosenbach M, Lipoff JB, Moustafa $D$, Tyagi A, et al. Cutaneous reactions reported after Moderna and Pfizer COVID-19 vaccination: a registry-based study of 414 cases. J Am Acad Dermatol 2021; 85: 46-55. 